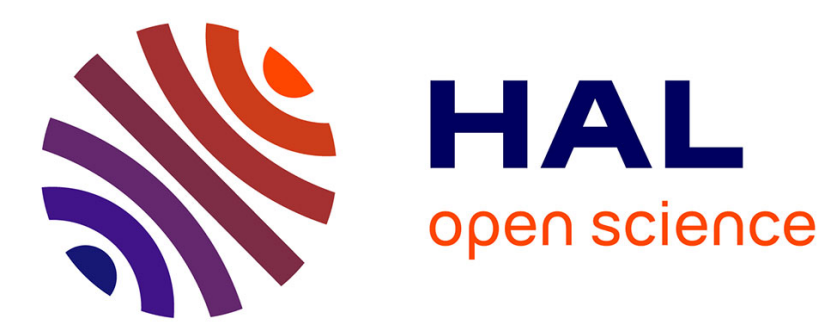

\title{
Touchée par le féminisme. l'Antiquité avec les sciences humaines
}

\author{
Violaine Sebillotte Cuchet
}

\section{To cite this version:}

Violaine Sebillotte Cuchet. Touchée par le féminisme. l'Antiquité avec les sciences humaines. P. Payen, E. Scheid-Tissinier. Anthropologie de l'Antiquité. Anciens objets, nouvelles approches, Brepols, pp.143-172, 2013, 978-2-503-54697-1. halshs-01853884

\section{HAL Id: halshs-01853884 https://shs.hal.science/halshs-01853884}

Submitted on 5 Aug 2018

HAL is a multi-disciplinary open access archive for the deposit and dissemination of scientific research documents, whether they are published or not. The documents may come from teaching and research institutions in France or abroad, or from public or private research centers.
L'archive ouverte pluridisciplinaire HAL, est destinée au dépôt et à la diffusion de documents scientifiques de niveau recherche, publiés ou non, émanant des établissements d'enseignement et de recherche français ou étrangers, des laboratoires publics ou privés. 
ANTIOUITÉ ET SCIENCES HUMAINES LA TRAVERSEE DES FRONTIERES

\section{ANTHROPOLOGIE DE L'ANTIQUITE \\ ANCIENS OBJETS, \\ NOUVELLES APPROCHES}

édité par

Pascal Payen et Évelyne Scheid-Tissinier

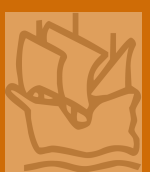

BREPOLS 


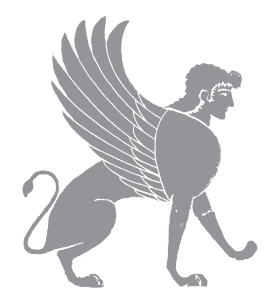

\section{ANTIOUITÉ ET SCIENCES HUMAINES \\ LA TRAVERSÉE \\ DES FRONTIERES}

\section{DIRECTEURS DE COLLECTION \\ Corinne BONNET \\ Pascal PAYEN \\ COMITÉ SCIENTIFIQUE \\ Zainab BAHRANI \\ (Columbia University, New York) \\ Nicola Cusumano \\ (Università degli Studi di Palermo) \\ Erich GRUEN \\ (University of California, Berkeley) \\ Nicholas PurcelL \\ (St John's College, Oxford) \\ Aloys WinTERLing \\ (Humboldt Universität, Berlin)}

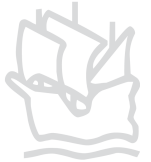




\title{
Anthropologie de l'Antiquité
}

Anciens objets, nouvelles approches

\author{
Édité par \\ PASCAL PAYEN \\ ET ÉVELYNe SCHEID-TiSSINIER
}

政

BREPOLS 
(C) 2012 Brepols Publishers n.v., Turnhout, Belgium

All rights reserved.

No part of this publication may be reproduced, stored in a retrieval system, or transmitted, in any form or by any means, electronic, mechanical, photocopying, recording, or otherwise, without prior permission of the publisher.

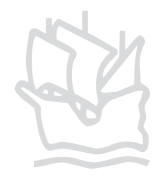

D/2012/0095/152

ISBN 978-2-503-54697-1

Printed in the E.U. on acid-free paper 


\section{SOMMAIRE}

Évelyne SCHEID-TISSINIER

Introduction

\section{ÉCHANGES ET TRANSFERTS}

Vincent AzOULAY

Du paradigme du don à une anthropologie pragmatique de la valeur

Ton DeRks

Les rites de passage dans l'Empire romain :

esquisse d'une approche anthropologique

Nicholas Purcell

Quod enim alterius fuit, id ut fiat meum, necesse est aliquid intercedere (Varro). The Anthropology of Buying

and Selling in Ancient Greece and Rome: An Introductory Sketch

\section{IDENTITÉS ET REPRÉSENTATIONS}

\section{Corinne BONNET}

Lorsque les " autres " entrent dans la danse. Lectures phéniciennes des identités religieuses en contexte multiculturel

Pauline SCHMitT PANTEL

Les mours des Grecs : histoire, anthropologie et politique

Violaine Sebillotte Cuchet

Touchée par le féminisme. L'Antiquité avec les sciences humaines 
Maurizio BetTINI

Entre "émique » et "étique ».

Un exercice sur le Lar familiaris

\section{SCHÉMAS CULTURELS ET MODÈLES SOCIAUX}

Pascal PAYEN

Sur la violence de guerre en Grèce ancienne.

Anthropologie, histoire et structure

Adeline Grand-Clément

Poikilia. Pour une anthropologie de la bigarrure

Évelyne SCHEID-TISSINIER

Du bon usage des émotions dans la culture grecque

Emmanuelle VALETte

La voix des monuments, l'écriture du carmen :

l'élégie romaine entre histoire, littérature

et anthropologie du monde romain

Andreas WitTenburg

Antiquité et anthropologie en Allemagne:

Eduard Meyer et après

BIBLIOGRAPHIE

SYNTHÈSES / ABSTRACTS

INDEX THÉMATIQUE

INDEX DES AUTEURS

INDEX DES NOMS 


\section{VIOLAINE SEBILLOTTE CUCHET \\ TOUCHÉE PAR LE FÉMINISME. L'ANTIQUITE AVEC LES SCIENCES HUMAINES}

La plupart des équipes de recherches françaises de sciences humaines intègrent aujourd'hui, d'une manière ou d'une autre, la question des femmes, du féminin ou plus largement celle du genre $^{1}$. Les recherches et les questionnements issus des mouvements féministes du second $\mathrm{xx}^{\mathrm{e}}$ siècle ont, semble-t-il, convaincu la plupart des chercheurs - les chercheuses étant aussi devenues plus nombreuses - de la nécessité de rendre compte de la présence des femmes, comme de celle des hommes, dans toutes les sociétés humaines. Bien davantage qu'un effet de mode, par définition passager, le féminisme a également marqué de son empreinte l'anthropologie de l'Antiquité comme les autres branches des sciences sociales.

En évoquant dans le titre de cette contribution une anthropologie de l'Antiquité touchée par le féminisme, je fais certes référence au mouvement social et politique - moderne et occidental - qui a lutté pour l'égalité des droits des femmes, mais je fais également référence à la conception aujourd'hui consensuelle de l'Homme (anthrôpos) comme individu concret que décrit la sociologue Irène Théry ${ }^{2}$. Doté de caractéristiques incarnées, l'Homme n'est plus cet individu abstrait et neutre du point de vue du sexe - ce qui est une manière de masquer le point de vue masculin. De la nou-

${ }^{1}$ Je tiens à remercier Sandra Boehringer pour sa lecture critique et instructive d'une première version de cet article et, d'une façon générale, pour le temps passé à échanger avec moi sur ce sujet.

${ }^{2}$ I. ThÉRY, «Avant-propos ", in I. ThÉRY, P. Bonnemère (éd.), Ce que le genre fait aux personnes, Paris, 2008, p. 7-11. 
velle perspective anthropologique, le point de vue du chercheur sort considérablement modifié : il intègre désormais la variété des possibles de l'existence humaine, le sexe en constituant une dimension parmi d'autres ${ }^{3}$.

Les relations entre histoire ancienne, anthropologie et histoire des femmes ont été analysées à plusieurs reprises ${ }^{4}$. Si je devais prolonger aujourd'hui une analyse rétrospective sur les outils, les méthodes et les questionnements, j'ajouterais que les années 2000 ont été dominées par la critique des catégories d'interprétation, en premier lieu la catégorie de $\operatorname{sex}^{5}$. Pour certains, cette critique a conduit à un aggiornamento salutaire ${ }^{6}$. C'est ce tournant et les raisons de ce tournant que je voudrais analyser. Cela me permettra de mettre en avant les directions de recherches qu'une anthropologie de l'Antiquité en dialogue avec les sciences humaines et sociales peut développer. Dans le cadre d'une seule contribution, il n'est pas question de proposer autre chose qu'une lecture générale de la perspective qui a été adoptée par l'anthropologie de l'Antiquité et des références qui ont marqué l'évolution de la problématique. Ce texte ne peut être exhaustif et, bien évidemment, il ne reflète pas la totalité des approches et des travaux qui ont été menés depuis les débuts de l'anthropologie historique de l'Antiquité. Il s'attache aux grandes tendances qui, selon moi, se sont développées en France, souvent en relation avec des travaux américains, lesquels connaissent une réception plus importante que d'autres travaux en langues étrangères, non traduits ou mal compris. Quant aux directions de recherche, ce sont celles dont je connais le développement et qui me sont les plus familières.

${ }^{3}$ Les sociologues utilisent l'expression d'intersectionnalité lorsqu'il s'agit de considérer l'ensemble des interactions sociales : L. BÉRÉNI, S. CHAUvin, A. JAUNAIT et alii, Introduction aux Gender Studies, Paris, De Boeck, 2008, p. 211-215.

${ }^{4}$ Pour le point de vue français, voir P. Schmitt Pantel, "La Différence des sexes. Histoire, anthropologie et cité grecque ", in M. Perrot (éd.), Une histoire des femmes est-elle possible?, Marseille, 1984, p. 98-119 et 223-225 [article repris dans P. Schmitt Pantel, Aithra et Pandora. Femmes, Genre et Cité dans la Grèce antique, Paris, L'Harmattan, 2009, p. 23-37f; P. Schmitt PANTEL, « L'Histoire des femmes en histoire ancienne aujourd'hui ", in G. Duby, M. Perrot (éd.), Histoire des femmes, 5 vol., Paris, $1991^{2}$ (1990), vol. I : L'Antiquité, p. 493-502 [article repris dans Schmitt Pantel, Aithra et Pandora, p. 39-48].

${ }^{5}$ V. Sebillotte Cuchet, "Les Antiquistes et le Genre ", in V. Sebillotte Cuchet, N. Ernoult (éd.), Problèmes du genre en Grèce ancienne, Paris, 2007, p. 11 26, en part. p. 17-18.

${ }^{6}$ THÉRY, «Avant-propos », p. 8. 
Je propose, en premier lieu, de rappeler comment la première anthropologie historique de l'Antiquité, qui s'est développée en France avec Louis Gernet, a été marquée par la perspective masculine caractérisant l'ensemble des sciences sociales de son époque, et comment la recherche sur le genre, dans les années 1980-1990, a permis que la différence des sexes soit intégrée dans les approches en histoire ancienne. J'analyserai ensuite la manière dont la réflexion a buté, dans les mêmes années, sur cette notion, en en figeant les interprétations. Enfin, je montrerai pourquoi l'anthropologie de l'Antiquité est appelée à inscrire à son programme une question nouvelle et devenue centrale pour les sciences sociales, une question que je formulerais ainsi : quelles places les sociétés font-elles, ou ont-elles faite, à la différence des sexes ? De la reconnaissance de la différence des sexes dans l'histoire à l'histoire de la différence des sexes, la perspective a radicalement changé 7 .

\section{L'intégration de la différence des sexes dans l'écriture de l'histoire}

Comme chacun sait, l'alliance de l'anthropologie et des sciences de l'Antiquité doit essentiellement à Jean-Pierre Vernant et à Louis Gernet, considéré par Jean-PierreVernant comme le père du courant historiographique désigné dans les années 1970 par l'expression $d^{\prime}$ " anthropologie historique de l'Antiquité ${ }^{8}$ ». Jean-Pierre Vernant souligne l'apport du savant dans la préface qu'il rédige pour la publication du livre Anthropologie de la Grèce antique. Le recueil rassemble divers articles de Louis Gernet, publiés auparavant dans des revues savantes et réunis par Jean-Pierre Vernant en 1968.

Qu'importaient les thèmes ? Quels qu'ils fussent, Gernet était à son affaire, dans son sujet, parce qu'il était chez lui en Grèce ancienne, à la façon d'un ethnologue qui, parti dès l'âge d'homme explorer une terre lointaine, ne l'aurait plus

${ }^{7}$ Le titre retenu pour un ouvrage collectif d'histoire des femmes et du genre paru en 2010 est significatif: M. Riot-SARCEY (éd.), De la différence des sexes : le genre en histoire, Paris, Larousse, 2010.

${ }^{8}$ Sur les rapports entre anthropologie et histoire, de manière générale, voir A. Bensa, "Anthropologie et histoire " dans Delacroix, Historiographies, p. 4253 , qui situe la mutation de l'histoire vers une histoire anthropologique dans les années 1920. 
jamais quittée et en comprendrait le peuple à la fois du dedans et du dehors, avec le double regard de l'indigène et de l'étranger'.

La démarche adoptée par Louis Gernet, qui se désigne comme un " anthropologue ${ }^{10}$ ", est totalisante, reconnaissant l'héritage du sociologue et ethnologue Marcel Mauss : il s'agit, comme le rappelle encore Jean-Pierre Vernant, de

partir des réalités collectives, à tous les niveaux, en cerner la forme dense, en bien mesurer le poids social, mais ne jamais les séparer des attitudes psychologiques, des mécanismes mentaux sans lesquels ni l'avènement ni la marche, ni les changements des institutions ne sont intelligibles ${ }^{11}$.

Pourtant, à observer de plus près, le regard est alors très ciblé et les femmes laissées de côté.

\subsection{L'anthropologie historique : les femmes de côté}

Les thématiques privilégiées par les anthropologues de l'Antiquité sont les institutions et le droit, la religion, l'économie et les échanges (par l'étude du don notamment) ${ }^{12}$. Les femmes apparaissent dans l'Anthropologie de la Grèce antique à propos de la religion. Elles sont dotées d'une forte valeur symbolique, pour l'homme s'entend, laquelle est interprétée en terme d'altérité. À l'occasion du compte rendu du livre d'Henri Jeanmaire sur Dionysos ${ }^{13}$, paru en 1951, Louis Gernet souligne l'importance des femmes dans les rituels, organisés, dit-il, selon la différence des sexes :

9 J.-P. Vernant, «Préface » à L. Gernet, Anthropologie de la Grèce antique, Paris, Flammarion, $1982^{2}$ (1968), en part. p. 6.

${ }^{10}$ L. GER NET, «L'Anthropologie dans la religion grecque » (1955), in GERNET, Anthropologie, p. 13-27, en part. p. 13.

${ }^{11}$ Vernant, «Préface ", p. 7-8.

${ }^{12}$ L'anthropologie de l'Antiquité est peu sensible à la question des femmes et de la différence des sexes, ou la considère comme non suffisamment documentée : M.I. FInLey, Les Anciens Grecs. Une introduction à leur vie et à leur pensée, Paris, Maspéro, $1979^{3}$ (1963), en part. p. 131-133 ; S.C. Humphreys, Anthropology and the Greeks, London, Routledge, $2004^{3}$ (1978), développe les thématiques des échanges, de l'économie et du droit. Même son analyse de la parenté n'intègre que très peu les relations de sexe (p. 193-208).

${ }^{13}$ H. Jeanmaire, Dionysos. Histoire du culte de Bacchus, Paris, Payot, 1951. 
Ce qui a toujours frappé, en effet, c'est l'importance de l'élément féminin dans cette religion. Les égarements et la frénésie que désigne le mot d'orgiasme, c'est le plus souvent des femmes qui y sont sujettes; dans les représentations figurées, nous ne voyons guère qu'elles aux moments pathétiques du culte. Comment comprendre cette donnée ? Il est bien connu que la nature féminine fournit le «terrain favorable ». [...] On peut comprendre, dans le dionysisme, le rôle prééminent de la femme parce que cette valeur essentielle [i.e. l'évasion, caractéristique du dionysisme selon Jeanmaire et Gernet], la femme est mieux faite pour l'incarner. Elle est moins engagée, moins intégrée. Elle est appelée à représenter, dans la société, un principe qui s'oppose à la société ellemême - et dont celle-ci a pourtant besoin. Il faut croire que ce besoin, sur le plan religieux, a été ressenti par les Grecs avec acuité ${ }^{14}$.

L'analyse est nuancée sur le plan des informations : il y a aussi des acteurs masculins, dit Louis Gernet, dans le culte de Dionysos ${ }^{15}$. Néanmoins est affirmée une idée forte qui va marquer la recherche dans la seconde moitié du $\mathrm{XX}^{\mathrm{e}}$ siècle : les femmes représentent une valeur d'altérité du fait de leur caractère quasi asocial. Comme les savants de sa génération, Gernet produit une anthropologie qui considère que les femmes constituent à la fois un ensemble homogène et une catégorie à part ${ }^{16}$.

\subsection{Le tournant des années 1980 :}

les femmes intégrées dans l'histoire globale

On le sait bien, ce sont des chercheuses qui ont ouvert la brèche en soulignant la place jouée par les femmes dans l'organisation des sociétés. Le rôle d'Annette Weiner, pionnière de la nouvelle anthropologie féministe, a été abondamment souligné même s'il ne doit pas faire oublier les tentatives isolées qui ont précédé ou

${ }^{14}$ L. Gernet, « Dionysos et la religion dionysiaque : éléments hérités et traits originaux » (1953), in GeRnet, Anthropologie, p. 110-111.

${ }^{15}$ Intuition qui a été plus tard étayée par A.-F.JAcCottet, Choisir Dionysos. Les associations dionysiaques ou la face cachée du dionysisme, Zürich, Akanthus, 2003, p. 65-94.

${ }^{16}$ Voir l'analyse de Josine Blok sur cette historiographie qui a isolé les femmes (conçues comme un groupe homogène) de la vie sociale en les dotant d'une essence différente de celle des hommes : "Sexual Asymmetry. A Historiographical Essay ", in J. Blok, P. Mason, Sexual Asymmetry, Amsterdam, 1987, p. 1-57, en part. p. 1-37. 
l'ont accompagnée ${ }^{17}$. Son travail, sur les pas de Malinovski, a radicalement modifié le regard des historiennes et des anthropologues qui avaient adopté la perspective féministe. Répétons-le : il s'agissait pour elle, en partant d'un des "lieux saints de l'anthropologie ", les îles Trobriand, de considérer les relations entre hommes et femmes pour elles-mêmes au lieu de considérer les femmes comme des éléments périphériques dans la structuration des sociétés. Le résultat fut de souligner les domaines où l'autorité et la valeur sociale des femmes étaient reconnues ${ }^{18}$. Comme l'écrit Pauline Schmitt Pantel, l'article d'Annette Weiner, publié en 1982 dans les Annales et qui réclame que la question de la différence des sexes devienne un objet d'étude pour anthropologues au même titre que « la parenté, le politique ou l'économique ", correspond à une prise de conscience simultanée dans toutes les sciences sociales, histoire incluse : en 1979, Arlette Farge réclamait également que le silence soit levé sur le "partage entre le masculin et le féminin ${ }^{19}$ " et, dès 1973, Sarah Pomeroy dressait le bilan des connaissances pour l'Antiquité classique ${ }^{20}$. Les publications sont alors prolifiques, ce dont témoigne le catalogue bibliographique en ligne Diotima, lui-même non exhaustif ${ }^{21}$.

${ }^{17}$ A. Weiner, Women of Value, Men of Renown: New Perspectives in Trobriand Exchange, Austin - London, University of Texas Press, 1976 et A. WeInER, «Plus précieux que l'or : relations et échanges entre hommes et femmes dans les sociétés d'Océanie ", Annales ESC 37/2 (1982), p. 222-245. La revue Annales ESC publie cet article dans un dossier plus large intitulé Masculin-féminin.

${ }^{18}$ I. THÉRY, « Pour une anthropologie comparative de la distinction de sexe », in ID., La Distinction de sexe. Une nouvelle approche de l'égalité, Paris, 2007, p. 15-43, et part. p. 27-28.

${ }^{19}$ WeINER, «Plus précieux que l'or》, p. 222 et A. FAR GE, L'Histoire sans qualités, Essais, Paris, Éd. Galilée, 1979, p. 18 ; tous deux cités par Schmitt PAntel, Aithra et Pandora, p. 23-24.

${ }^{20}$ S.B. Pomeroy, «Selected Bibliography on Women in Antiquity ", Arethusa 6 (1973), p. 127-155, avant son ouvrage, Goddesses, Whores, Wives and Slaves:Women in Classical Antiquity, New York, Schocken Books, 1975, qui mettait en avant la pauvreté des informations concernant les femmes dans l'Antiquité (p. 226-236), un constat qui est discutable (voir BLOK, "Sexual Asymmetry », p. 1-57).

${ }^{21}$ Diotima. Materials for the Study of Women and Gender in the Ancient World (www. stoa.org/diotima). La base de données n'a pas été mise à jour depuis novembre 2006 (site consulté le 20 janvier 2011). 


\subsection{L'histoire des femmes de l'Antiquité à aujourd'hui}

Aujourd'hui, l'Antiquité comme toutes les autres périodes de l'histoire bénéficie de cet éclairage, féministe à l'origine, qui balaie plus généralement l'ensemble des sciences sociales. L'attention s'est d'abord orientée vers l'étude de femmes en situation de pouvoir. Après le travail pionnier de Grace H. Macurdy ${ }^{22}$, les études d'Elizabeth Carney sur les reines et les dynastes féminines de Macédoine puis de Carie ont précisé les modalités de leurs interventions dans des sphères de compétence jusque là perçues comme uniquement masculines : ainsi l'octroi de privilèges, la décision de lever une armée, voire la participation aux combats ${ }^{23}$. Cette direction de recherche est particulièrement féconde pour les périodes hellénistique et romaine ${ }^{24}$. D'autres recherches ont porté sur la situation des femmes dans la cité classique, souvent Athènes au IV siècle, en tentant d'orienter le regard vers des femmes moins exceptionnelles ${ }^{25}$. L'étude de Lin Foxhall sur la loi et le discours judicaire, en particulier à propos du Contre Aphobos de Démosthène, me parait, de ce point de vue, fondamentale ${ }^{26}$. En considérant une famille citoyenne dans sa globalité, le défunt (le père de Démosthène), la mère (Cléoboulé), le fils (Démosthène), les tuteurs (Aphobos, Démophon, Therippidès), Lin Foxhall montre comment chacun, selon des modalités qui lui sont propres, agit et réagit face aux autres et comment, finalement, le point de vue de la mère, Cléo-

${ }^{22}$ G.H. Macurdy, Vassal-Queens and Some Contemporary Women in the Roman Empire, Baltimore, Johns Hopkins Press, 1937.

${ }^{23}$ E.D. Carney, Women and Monarchy in Macedonia, Norman, University of Oklahoma Press, 2000 et E.D. CARney, "Women and Dunasteia in Caria ", American Journal of Philology 126/1 (2005), p. 65-91.

${ }^{24}$ Par exemple : R.VAn Bremen, The Limits of Participation. Women and Civic Life in the Greek East in the Hellenistic and Roman Periods, Amsterdam, Gieben, 1996, en part. p. 16-19 ; F. Bertholet, A. Bielman Sanchez, R. Frei-Stolba (éd.), Egypte - Grèce - Rome, les différents visages des femmes antiques, Bern, Peter Lang, 2008 ; F. CENER INI (éd.), Dive e donne: mogli, madri, figlie e sorelle degli imperatori, da Augusto a Commodo, Imola, Angelini, 2009 ; A. KolB (éd.), Augustae. Machtbewusste Frauen am römischen Kaiserhof ?, Berlin, Akademie-Verlag, 2010.

${ }^{25}$ On peut citer les travaux portant sur l'archéologie et l'iconographie funéraire ; ainsi, parmi d'autres : R. OsBorne, « Funerary Monuments, the Democratic Citizen and the Representation of Women ", in F.R. Adrados, L. Beschi, W. BurkerT (éd.), Colloque international Démocratie athénienne et culture, Athènes, 1996, p. 229-242.

${ }^{26}$ L. Foxhall, "The Law and the Lady: Women and Legal Proceedings in Classical Athens ", in L. Foxhall, A.D. Lewis (éd.), Greek Law in its Political Setting, Oxford, 1996, p. 133-152. 
boulé, une veuve placée par voie testamentaire sous la tutelle de plusieurs kurioi, parvient à s'imposer dans la pratique et dans le droit. La tutelle (kureia), loin de rendre les femmes athéniennes passives, constituait certes un obstacle à surmonter - Cléoboulé doit patienter jusqu'à la majorité de son fils pour que son point de vue l'emporte enfin au tribunal - mais elle ne signifiait pas que les divers acteurs sociaux tenaient les Athéniennes pour insignifiantes. L'appréciation de la tutelle pesant sur les Athéniennes, de même que l'appréciation de l'effectivité de la loi, est devenue un enjeu central dans les recherches actuelles ${ }^{27}$. Le regard aujourd'hui porté sur les pratiques poétiques et culturelles de la Grèce archaïque est rafraîchi dans une perspective féministe :l'épopée, longtemps tenue pour le genre littéraire masculin par excellence, est interrogée du point de vue des femmes. Lillian Doherty, sensible aux études menées par les ethnologues qui travaillent sur l'Inde moderne, envisage, avec d'autres ${ }^{28}$, la possibilité d'un auditoire féminin qui aurait influencé le contenu des œuvres récitées dans les cours et les palais des aristocraties grecques. Des commanditaires féminines expliqueraient certaines thématiques de la poésie épique archaïque, en particulier la glorification d'héroïnes en série ${ }^{29}$.

Les enquêtes menées à partir d'exemples de femmes en position particulières (reines, dynastes, évergètes, Athéniennes, hérö̈nes épiques, etc.) ont amené les chercheurs à repenser les faits sociaux

${ }^{27}$ Des kurioi différents peuvent être choisis en fonction des affaires à traiter (C. VIaL, "Statut et subordination », in O. CAvalier [éd.], Silence et fureur. La femme et le mariage en Grèce. Les antiquités grecques du Musée Calvet, Avignon, 1996, p. 339-358, en part. p. 355) et la tutelle ne s'exercer que sur des aspects très restreints de la vie sociale (S. Hodkinson, Property and Wealth in Classical Sparta, London - Swansea, Duckworth, 2000, p. 102-103 et S. Hodkinson, « Female Property Ownership and Empowerment in Classical and Hellenistic Sparta ", in T.J. Figueira [éd.], Spartan Society, Swansea, 2004, p. 103-136).

${ }^{28}$ Là encore il ne s'agit pas d'être exhaustif, du moins notons le collectif : E. Greene (éd.), Reading Sappho: Contemporary Approaches, Berkeley, University of California Press, 1996 ainsi que les travaux de John Winkler : J.J. WinkLER, Désirs et contraintes en Grèce ancienne, Paris, EPEL, $2005^{2}$ (1990), en part. p. 247-352.

${ }^{29}$ L. Doherty, Siren Songs: Gender, Audiences, and Narrators in the Odyssey, Ann Arbor, University of Michigan Press, 1995 ; L. DOHERTY, « Putting the Women Back into the Hesiodic Catalogue of Women ", in V. ZajKo, M. LeONARD (éd.), Laughing with Medusa. Cassical Myth and Feminist Thought, Oxford, 2006, p. 297-325 et « La "Maternité" de l'Odyssée. Fortune d'un fantasme victorien ", Clio HFS 32 (2010), p. 149-164. L. Doherty s'appuie en particulier sur l'article de V. NARAYANA RaO, "A Ramayana of their Own:Women's Oral Tradition in Telugu ", in P. Richman (éd.), Many Ramayanas, Berkeley, 1991, p. 114-136. 
dans lesquels ces femmes intervenaient : le pouvoir dynastique, le pouvoir civique et économique dans le cadre familial, la tutelle et la domination masculine dans la sphère juridique, la production poétique, etc. Ces faits apparaissent alors dans toute leur complexité : le pouvoir royal prend des formes diversifiées, les domaines relevant de la tutelle semblent se restreindre ou dépendre de certains types de pratiques de négociation des conflits, etc. On peut également faire le chemin dans l'autre sens : partir d'un fait social, comme la citoyenneté classique ${ }^{30}$, et le relire d'un point de vue mixte, en intégrant les femmes. Après Claudine Leduc ${ }^{31}$, entre autres, c'est la voie suivie aujourd'hui par Josine Blok ${ }^{32}$. Selon elle, le critère de la participation aux magistratures que nous tenons, depuis Aristote, pour la définition de la citoyenneté, est une proposition a minima faite par le philosophe dans le but de construire une argumentation logique sur la cité juste. Cette définition n'aurait donc que peu à voir avec ce que les Athéniens percevaient de la citoyenneté. En reprenant les discours tenus dans les procès ainsi que les décrets publics, Josine Blok démontre que la citoyenneté est une réalité familiale, impliquant des citoyens et des citoyennes dans une relation collective établie avec les dieux. Les études sur les femmes aboutissent ainsi à la remise en cause de ce qui a long-

${ }^{30}$ La question de la citoyenneté a depuis longtemps été perçue comme un enjeu pour l'histoire des femmes : C. Mossé, «L'Antiquité. Lecture critique du tome I de l'Histoire des femmes ", in G. Duby, M. Perrot (éd.), Femmes et Histoire, Colloque de la Sorbonne (13-14 novembre 1992), Paris, Plon, 1993, p. 1924, en part. p. 23-24. Voir également, C. Patterson, " Hai Attikai:The Other Athenians ", in M. Skinner (éd.), Rescuing Creusa: New Methodological Approach to Women in Antiquity, Helios n.s. 13/2 (1987), p. 49-67, en part. p. 54-55.

${ }^{31}$ C. Leduc, "Comment la donner en mariage ? ", in G. Duby, M. Perrot (éd.), Histoire des femmes en Occident, Paris, 1991, vol. I : L'Antiquité, p. 259-316. À cet important article, il faut ajouter les publications ultérieures de Claudine Leduc dont l'apport est résumé par P. SCHMITT PANTEL, "L'Histoire des femmes ", in Schmitt Pantel, Histoire des femmes, p. 603-614, en part. p. 612.

32 J. BLOK, "Recht und Ritus der Polis. Zu Bürgerstatus und Geschlechterverhältnissen im klassischen Athen ", Historische Zeitschrift 278/1 (2004), p. 1-26 ; "Becoming Citizens. Some Notes on the Semantics of "Citizen" in Archaic Greece and Classical Athens ", Klio 87 (2005), p. 7-40 et "Perikles' Citizenship Law: A New Perspective » Historia 58 (2009), p. 141-170.Voir également les deux conférences qu'elle a données les 11 et 25 janvier 2011 à Paris (INHA) sous le titre : Définitions de la citoyenneté : proposition de révision et Pratiques de la citoyenneté : descendance, timai et archai. Ces conférences annoncent la sortie prochaine de son ouvrage Citizenship, Cult and Community, Cambridge, Cambridge University Press. Voir également, sur l'ethnique Athenaia, A. JACQUEMIN, «Un autre conte de deux cités ou...Athéniennes et fières de l'être ", Ktèma 30 (2005), p. 337-347. 
temps été tenu pour une caractéristique fondamentale des structures sociales et politiques de la Grèce des cités : l'exclusion des femmes du politique.

Il est très peu probable que l'on en revienne un jour à l'époque d'avant Annette Weiner, à l'époque d'avant Arlette Farge, à l'époque d'avant Sarah Pomeroy, celle d'une histoire masculine ignorant son point de vue. Les acquis scientifiques de la révolution féministe des années 1960-1970 sont patents : à la fois l'organisation sociale et l'imaginaire (ou les représentations) articulent des rapports de sexe et des constructions culturelles du féminin et du masculin, qui, de ce fait, n'échappent pas à l'histoire.

\section{La question du genre et la différence des sexes comme "butoir 》 de la pensée}

Le tournant que fut l'introduction du gender, traduit par "genre " dans les années 1980-1990 en France, a donné un fort élan aux recherches sur les femmes mais également sur les hommes en tant qu'hommes, et ceci dans l'ensemble des sciences sociales. De ce point de vue, l'anthropologie de l'Antiquité n'a pas été la dernière à suivre la voie ouverte aux Etats-Unis : à la fois le collectif italien Le donne in Grecia et le premier volume de l'Histoire des femmes en occident témoignent de l'intérêt porté par les spécialistes de l'Antiquité (et, précisons-le, par un éditeur italien audacieux) aux outils heuristiques importés des autres sciences sociales ${ }^{33}$. À la fin des années 1990 cependant, l'outil semble, pour certains, avoir déjà épuisé ses promesses : aux yeux d'un certain nombre de féministes, le genre n'a pas permis d'échapper à la problématique de la différence, laquelle ne semble pas pouvoir être pensée autrement qu'à l'intérieur d'un rapport de domination des hommes sur les femmes. Les thèses de Nicole Löraux d'une part et de Françoise Héritier d'autre part jouent alors un rôle crucial dans l'orientation de la recherche.

${ }^{33}$ G. ArRigoni, «Le donne dei "margini" e le donne "speciali" ", in G. ArRIGONI (éd.), Le donne in Grecia, Roma - Bari, $2008^{2}$ (1985), p. XVI-XVII, qui n'emploie pas le terme de " genre " et Schmitt Pantel, "L'Histoire des femmes ", p. 493-502, en part. p. 494-498. 


\subsection{Les ambitions du genre}

Le genre, dans sa version française, signifie le sexe social c'est-à-dire l'ensemble des assignations et des représentations associées au sexe, féminin ou masculin ${ }^{34}$. La perspective de genre est directement issue des travaux de Simone de Beauvoir dénaturalisant "l'éternel féminin » et de ceux de Margaret Mead s'interrogeant sur la variabilité de la définition de la femme ${ }^{35}$. Les études de genre visent alors à repérer les caractéristiques du féminin et celles du masculin, dans telle ou telle société, telle ou telle culture. L'anthropologie dans sa dimension historique est particulièrement sensible aux évolutions de ces représentations dans le temps, lesquelles sont étudiées dans des corpus précis, dans les traités techniques médicaux, dans la production théâtrale attique, dans l'épigraphie funéraire, etc. ${ }^{36}$

Le genre est alors une manière, pour les chercheurs et les chercheuses, de penser la construction des identités hommes/femmes de façon relationnelle et surtout non séparée ${ }^{37}$. La notion ne se conçoit pas sans une réflexion sur le rapport de domination des hommes sur les femmes. La problématique générale est donc celle de la domination masculine, une domination tenue pour quasiment structurelle même si elle varie dans les formes ${ }^{38}$. Le terme, venu des sociologues, est peu employé par les historiennes qui préferent

${ }^{34}$ Sur cette définition et ses avantages, de même que sur les différentes expressions utilisées, voir la présentation qu'en fait Schmitt Pantel, "L'Histoire des femmes ", p. 493-502, en part. p. 496-498.

${ }^{35}$ S. De Beauvoir, Le Deuxième Sexe, Paris, Gallimard, $2000^{5}$ (1949), vol. I, p. 13 ; M. MeAD, L'Un et l'autre sexe, Paris, Gonthier, $1988^{3}$ (1948), en part. p. 420 : "Les enfants apprennent au moyen de leur corps et à la manière dont il est traité par les autres, qu'ils sont du sexe masculin ou féminin. » Margaret Mead avait déjà écrit son célèbre livre From the South Seas, Studies of Adolescence and Sex in Primitive Societies, New York, W. Morrow, 1939, qui a été traduit par Georges Chevassus sous le titre Moeurs et sexualité en Océanie, Paris, Plon, 1963.

${ }^{36}$ La bibliographie est très importante : on peut se référer à P. SCHMitT PANTeL, L. Bruit, "L'Historiographie du genre : état des lieux », in Sebillotte Cuchet, ERnOult, Problèmes du genre, p. 27-48, comme aux diverses contributions contenues dans ce volume qui présentent chacune un état des lieux limité au domaine analysé.

${ }^{37}$ Schmitt Pantel, "L'Histoire des femmes ", p. 493-502, en part. p. 502. Pour les anthropologues, voir le colloque After the Second Sex: New Directions, qui s'est tenu en avril 1984 à l'université de Pennsylvanie à l'initiative de Peggy R. Sanday : P.R. SAnDAY (éd.), Beyond the Second Sex: New Directions in the Anthropology of Gender, Philadelphie, University of Pennsylvania Press, 1990.

${ }^{38}$ Cette invariance de la domination de sexe est rappelée par P.R. SANDAY, "Beyond the Second Sex ", in Sanday, Beyond the Second Sex, p. 1-19, en part. p.1-4. 
parler de hiérarchie, de rapports de domination de sexe, ou garder tout simplement le mot genre - un terme suffisamment vague pour contenir tous les aspects. L'expression d'asymétrie des sexes renvoie, dans l'historiographie, à la pensée du XIX siècle qui élabore l'idée des deux sphères, privée et publique, dotées de valeurs inégales ${ }^{39}$.

Cette approche de genre, constructiviste, est directement liée au type de sources utilisées par les Antiquistes. Comme l'ont rappelé toutes les recherches des années 1980-1990, les historiens et historiennes de l'Antiquité travaillent majoritairement sur des sources écrites. Ces sources n'ont pas fondamentalement changé de nature aujourd'hui, même si la part prise par les données de nature archéologique n'a cessé d'augmenter. Elles restent, comme avant, majoritairement produites par des hommes mais, ainsi qu'on l'a montré plus haut, cela n'entache en aucune façon la recherche en histoire des femmes : les documents, quel que soit leur auteur, continuent à délivrer, selon le contexte de leur production et celui de leur réception, des informations utiles sur les partages de pouvoir, de prestige, de richesse, et concernent à la fois des hommes et des femmes. Néanmoins, l'absence caractéristique de sources directement produites par les femmes a largement participé à l'orientation de l'historiographie vers une histoire des représentations, implicitement présentées comme masculines ${ }^{40}$. Si toute tentative d'écriture de l'histoire des femmes se heurte au filtre masculin, la tentation est grande de s'orienter vers l'écriture d'une histoire des seules représentations masculines du féminin. La tentation de penser le féminin comme la caractéristique des femmes et le masculin comme la caractéristique des hommes, ne l'est pas moins. Certes, depuis Louis Gernet, la différence des sexes est entrée dans l'histoire et les femmes ne sont plus considérées comme asociales mais, quoi que situées à l'intérieur de la société, elles continuent souvent, dans l'historiographie des années 1990, de représenter l'altérité. La différence des sexes, qui ne sépare plus

${ }^{39}$ Blok, «Sexual Asymmetry »; C. Sourvinou-Inwood, « Male and Female, Public and Private, Ancient and Modern ", in E.D. ReEder (éd.), Pandora.Women in Classical Greece, Baltimore, 1996, p. 111-120, montrent à quel point ces distinctions sont inadéquates pour l'Antiquité.

${ }^{40}$ P. Schmitt Pantel, "Un fil d'Ariane " in Schmitt Pantel, Histoire des femmes, p. 21-27, en part. p. 24-25 ; N. LORAux (éd.), La Grèce au féminin, Paris, Les Belles Lettres, $2003^{2}$ (1993), p. XI ; ArRigOnI, « Le donne », p. XVIII. 
ceux qui sont dedans et ceux qui sont dehors, qui ne se confond plus avec l'opposition du public et du privé, constitue toujours la ligne de faille qui divise fondamentalement la société. Beaucoup le pensent sans l'expliciter ${ }^{41}$. Nicole Loraux, elle, l'a théorisé : la différence des sexes est le référentiel métaphorique de la division politique.

\subsection{Nicole Loraux : la différence des sexes comme structure psychique et politique}

Occupant une place essentielle dans l'anthropologie de la différence des sexes, l'œuvre de Nicole Loraux est difficile à synthétiser en quelques lignes ${ }^{42}$. Essayons néanmoins de souligner les directions majeures dans le domaine qui nous intéresse. Comme la plupart des chercheurs de sa génération, Nicole Loraux est fortement influencée par la perspective structuraliste ouverte par l'anthropologie de Claude Lévi-Strauss, même si, en plein accord avec l'anthropologie historique développée par Jean-Pierre Vernant, elle y associe une forte dimension d'historicité ${ }^{43}$. Or, il me semble que ce qui rapproche particulièrement Nicole Loraux de l'anthropologie sociale de Lévi-Strauss est le rapport qu'elle entretient, comme lui, avec la psychanalyse.

La loi de l'échange organisé des femmes, clef d'interprétation ultime des sociétés selon Lévi-Strauss, s'accorde en effet, toujours selon Lévi-Strauss, avec une autre loi générale, l'interdit de l'inceste, c'est-à-dire la détermination du conjoint par son statut

${ }^{41}$ Nombre de travaux, portant sur les femmes, font comme si celles-ci constituaient encore un chapitre à part de l'histoire générale.

${ }^{42}$ Sur le rayonnement des travaux de N. Loraux : Les Voies traversières de Nicole Loraux, Espaces Temps Les Cahiers Clio HFS 87-88 (2005) et le colloque "Les Femmes, le féminin et le politique après Nicole Loraux » organisé par le RING, le Centre Louis-Gernet, l'équipe Phéacie (Paris I et Paris VII) et l'Université Paris VIII, en novembre 2007 à Paris (INHA), publié sur le site du Center for Hellenic Studies, Washington, sous la responsabilité de C. Darbo-Peschanski, N. Ernoult, S. Georgoudi,V. Sebillotte-Cuchet, E.Varikas : http://chs.harvard.edu/, classics@ issue 7.

${ }^{43}$ N. Loraux, Les Enfants d'Athéna. Idées athéniennes sur la citoyenneté et la division des sexes, Paris, Maspéro, 1981, s'ouvre par une référence à Lévi-Strauss, p. 8. Sur la revendication d'une histoire prenant en compte le changement et les ruptures : N. Loraux, "Repolitiser la cité » (1986) in N. Loraux, La Cité divisée, l'oubli dans la mémoire d'Athènes, Paris, 1997, p. 41-58. 
de descendance et par l'obligation de distinguer le "Même " de "l'Autre ${ }^{44}$ ». Dans cette démarche, les anthropologues orientent la recherche vers la découverte d'un sens caché, organisateur de l'ensemble de la société et logé dans une forme très structurée d'inconscient social, ou, pour le dire d'une façon très générale, d'imaginaire. Dans le fonctionnement des sociétés, éclairé par la loi de l'échange des femmes, celles-ci sont considérées à la fois comme des objets d'échange - les objets (passifs) par excellence de l'échange - et comme les éléments signifiant l'altérité : étant membre du groupe des Autres, ceux avec qui l'échange est permis, les femmes représentent l'Altéritét ${ }^{45}$. Les notions d'imaginaire ou d'inconscient ainsi que d'altérité - même travaillée par l'idée du mélange - sont absolument fondamentales dans la recherche de Nicole Loraux.

Nicole Loraux choisit de travailler la question de la différence des sexes à partir de documents spécifiques, les mythes dans leurs formes écrites ou iconographiques ${ }^{46}$. Les mythes sont saisis par Nicole Loraux comme des discours construits, réélaborés à partir de matériaux narratifs plus anciens, par une cité qui les " travaille " pour leur faire exprimer son propre " imaginaire ", voire ses " fantasmes $»^{47}$. Dans le premier chapitre des Enfants d'Athéna, Nicole Loraux analyse les mythes athéniens de l'autochtonie comme les variantes d'une même structure désignée par le nom de «mythe civique ", de "représentation de la cité " ou "d'imaginaire civique $"$ ". Cette structure révèle un argument majeur, selon Nicole Loraux : l'exclusion des femmes de la cité, voire leur négation. La thèse repose en grande partie sur la lecture que Nicole Loraux fait du mythe rapporté parVarron, lui-même cité par Saint Augustin, indiquant qu'à l'origine d'Athènes les femmes ont été délibérément interdites de transmettre leur nom à leurs enfants, de porter le

${ }^{44}$ C. Lévi-Strauss, Les Structures élémentaires de la parenté, Paris, PUF, 1947.

${ }^{45}$ LÉVI-STRauss, Les Structures élémentaires, p. 135. Sur la dimension inconsciente de la praxis sociale, voir aussi C. LÉvi-STRAuss, La Pensée sauvage, Paris, Gallimard, $2008^{3}$ (1962) (Bibliothèque de la Pléiade), p. 555-872, en part. p. 829-830.

${ }^{46}$ Voir à ce propos le compte rendu du livre de N. Loraux par Maria Daraki dans les Annales ESC 37/5-6 (1982), p. 795-799.

${ }^{47}$ N. LorauX, «L'Imaginaire des autochtones » (1979), in Loraux, Les Enfants d'Athéna, p. 7-25, en part. p. 15. Sur le "travail du mythe " dans la cité, notion qui éloigne le mythe d'une conception trop fonctionnaliste, voir N. LORAuX, « L'Autochtonie : une topique athénienne " (1979), in Loraux, Les Enfants d'Athéna, p. 69-70.

${ }^{48}$ Loraux, Les Enfants d'Athéna, p. 40, 45, 51. 
nom d'Athéniennes et de voter. Ces sanctions interviennent à la suite de leur vote unanime en faveur d'Athéna et au détriment de Poséidon, lors de la querelle divine pour l'Attique ${ }^{49}$. La thèse repose également sur l'analyse du syntagme, répété dans certaines oraisons funèbres athéniennes (le corpus des oraisons funèbres est le corpus de prédilection de Nicole Loraux ${ }^{50}$ ), selon lequel la cité est à la fois "mère et patrie ${ }^{51}$ ", mais surtout, chez Démosthène, "père et patrie ». Dans la mesure où la mère désigne la terre civique, la première expression signifie, selon Nicole Loraux, l'éviction de l'élément féminin, c'est-à-dire des mères humaines. La seconde expression est plus explicite : la mère est cette fois évincée du couple parental, lequel est présenté, toujours dans les oraisons funèbres, comme un modèle pour penser la cité.

En accentuant l'aspect patriarcal de l'idéologie athénienne, il s'agit certes alors d'éloigner le spectre de la Terre-Mère, principe longtemps tenu pour originaire dans la constitution des sociétés anciennes notamment sous la figure du matriarcat ${ }^{52}$. À ce principe, Nicole Loraux substitue une opération politique liée à un contexte discursif précis (les oraisons funèbres) : il s'agit de la féminisation du territoire politique auquel est attribuée une fonction maternelle (génération et trophê). Mais les conclusions de Nicole Loraux vont plus loin :

Ainsi, toutes les instances imaginaires de la cité s'accordent à réduire tendanciellement la place faite à la femme dans la polis : la langue lui refuse un nom, les institutions la cantonnent dans la maternité, les représentations officielles lui retireraient volontiers jusqu'au titre de mère ${ }^{53}$.

La femme est l'exclue du politique.

${ }^{49}$ Les femmes ont gagné et sont "vaincues dans leur victoire ": Varron, cité par Saint Augustin, De civitate dei, XVIII, 9; Loraux, Les Enfants d'Athéna, p. 121.

${ }^{50} \mathrm{~N}$. Loraux, L'Invention d'Athènes. Histoire de l'oraison funèbre dans la "cité classique ", Paris, Mouton, 1981.

${ }^{51}$ Loraux, Les Enfants d'Athéna, citant Lycurgue et Démosthène : p. 66 et p. 119-153, citant Platon, Lysias et Démosthène : p. 130-131.

${ }^{52}$ Loraux, Les Enfants d'Athéna, p. 60-61 ; N. Loraux, «Et l'on déboutera les mères ", in N. Loraux, Les Expériences de Tiresias : le féminin et l'homme grec, Paris, 1989, p. 219-232, en part. p. 225 ; N. LORAux, Né de la terre, mythe et politique à Athènes, Paris, Éd. du Seuil, 1996, p. 128-168.

${ }^{53}$ Loraux, Les Enfants d'Athéna, p. 131. Cette conclusion a été fortement critiquée dès 1987 : PAtTerson, "Hai Attikai », p. 52-62. 
Prolongeant l'analyse dans diverses études postérieures, Nicole Loraux considère la césure féminin-masculin comme la clef permettant de saisir le fonctionnement global de la polis. Le féminin, entendu comme altérité, est le signe du conflit (stasis) qui menace et de la division du corps idéalement uni de la cité. Puisque le politique se construit comme étant ce qui permet la résolution du conflit - la réparation de la division - le féminin incarne son " envers ${ }^{54}$ ». La question de la différence des sexes ne peut être reléguée dans la sphère du privé ou de la famille : elle est incluse dans le politique comme l'envers est inclus dans l'endroit d'une étoffe. Les travaux de Nicole Loraux soulignent le fait que les divisions réelles qui traversent la cité et menacent sa cohésion peuvent toutes être formulées par cette autre opposition qu'est la différence des sexes ${ }^{55}$.

Si on peut dire que sa lecture de la différence des sexes comme structurante pour penser le politique manifeste un héritage remontant à Claude Lévi-Strauss ${ }^{56}$, la manière dont Nicole Loraux aborde la question des identités individuelles relève d'une autre approche, davantage redevable à sa lecture de Freud qui avait mis en avant les composantes mixtes des identités psychiques, d'où l'importance, chez elle, de la notion de "mélange ${ }^{57}$ ". Néanmoins, pour Nicole Loraux, le rapport au sexe est l'invariant de l'histoire. Dans la mesure où sa recherche porte, dit-elle, sur «l'homme grec dans son rapport à l'autre ", il lui est nécessaire de discuter ce qui apparait « de façon récurrente ", assure-t-elle, cette

envie des hommes Grecs à laquelle il faut bien donner sa juste désignation d'“envie de grossesse": le désir de s'alourdir de sensations pénétrantes que leur intensité toute féminine

${ }^{54}$ Loraux, Tirésias, p. 7-26, en part. p. 22.

${ }^{55}$ Loraux, La Cité divisée, p. 24 : «[...] à l'étude de la cité une a succédé la réflexion sur la division des sexes, et (que) la division des sexes a subrepticement introduit la cité comme cité divisée.»

${ }^{56}$ Cette lecture fait autorité : à la même époque, P. VIDAL-NAQUET, «Esclavage et gynécocratie dans la tradition, le mythe, l'utopie ", in ID., Le Chasseur noir, Paris, $1991^{3}$ (1981), p. 267-288, en part. p. 269.

${ }^{57} \mathrm{~N}$. Loraux associe la pensée grecque du mélange à ce qu'elle lit chez Freud : Loraux, Tirésias, p. 7-26, en part. p. 13. Elle s'explique sur son lien à la psychanalyse : Loraux, Tirésias, p. 23-26. 
devrait précisément interdire au citoyen paradigmatiquement viril $^{58}$.

La différence des sexes est ainsi pensée comme le socle d'un fantasme précis, caractéristique d'un sexe et visant l'autre sexe. Ce postulat a été largement développé par Françoise Héritier dont les thèses ont également marqué l'anthropologie de la différence des sexes. Leur portée est bien plus importante car non limitée à un contexte historique spécifique.

\subsection{Françoise Héritier et la valence différentielle des sexes}

Françoise Héritier, héritière de Claude Lévi-Strauss et ethnologue reconnue pour ses travaux de terrain en Haute-Volta, des travaux portant sur les mécanismes de l'alliance dans les systèmes de parenté, a prolongé les hypothèses de Nicole Loraux d'un point de vue anthropologique "à vocation universaliste ", en introduisant l'expression devenue célèbre de "valence différentielle des $\operatorname{sexes}^{59}$ ». Françoise Héritier explique en effet le phénomène de la domination masculine par le déséquilibre qu'elle observe - et interprète - dans le processus de la reproduction sexuée : les femmes ont un rôle extrêmement important dans ce processus, dit-elle, et les hommes voudraient les en dessaisir. Françoise Héritier a formulé plus précisément la hantise des hommes : c'est la capacité exorbitante des femmes à produire des enfants des deux sexes qui leur est intolérable ${ }^{60}$. Le postulat central est l'idée que les hommes ont peur de la "puissance féminine " manifestée dans sa puissance maternelle ${ }^{61}$. À partir de l'observation que la grossesse est un privilège de femmes, l'anthropologue soutient que les hommes ont toujours pensé que les femmes fabriquaient seules les enfants, garçons et filles (et on retrouve le "Même » et

${ }^{58}$ LORaux, Tirésias, p. 24, reprenant une expression de Marie-Christine Pouchelle.

${ }^{59}$ F. Héritier, Masculin-Féminin : la pensée de la différence, Paris, Odile Jacob, 1996 et Masculin-Féminin, Paris, Odile Jacob, 2002, vol. II : Dissoudre la hiérarchie. Cette thèse a été réaffirmée dans un petit livre destiné aux enfants : F. HÉRITIER, La Différence des sexes, Paris, Bayard Editions, 2010, en part. p. 59-68. Sur la valeur universaliste de cette anthropologie, voir la discussion des hypothèses de F. Héritier par l'historienne Anne-Marie Sohn :A.-M. SoHn, « Françoise Héritier entre invariants et nostalgie du passé ", Mouvements 12 (2000), p. 101-110.

${ }^{60}$ Héritier, Masculin-Féminin, vol. II, p. 21.

${ }^{61}$ Héritier, Masculin-Féminin, vol. II, p. 23. 
«l'Autre "), et qu'ils en auraient conçu un fort ressentiment à leur égard, au point d'élaborer des stratégies pour les en dessaisir. C'est dans cette perspective qu'interviendraient les mythes autour de la maternité de la terre ${ }^{62}$.

Les débats qui ont suivi la publication de ces conclusions ${ }^{63}$ permettent de mieux cerner l'impasse dans laquelle tous ceux qui ont travaillé avec le genre ont été conduits. Elle n'est pas sans rapport avec ce qui a amené certains - dont Joan W. Scott ellemême - à considérer le genre comme une notion à la fois dépassée et contre-productive ${ }^{64}$. L'ambiguité rarement levée entre féminin et femmes, masculin et hommes, et la banalisation de la polarité masculin-féminin a incontestablement renforcé l'argument de la puissance symbolique de la différence des sexes ${ }^{65}$. Comme le formule Anne-Marie Sohn, la thèse de l'inégalité des sexes repose, chez Françoise Héritier, sur une "évidence " : celle de la différence observable des sexes. La différence physique (autrement dit biologique) des sexes est le socle concret qui justifie la différenciation symbolique du masculin et du féminin ${ }^{66}$. La thèse de l'insupportable

${ }^{62}$ Héritier, Masculin-Féminin, vol. II, p. 20 : « L'importance et la quasi-universalité de ces représentations qui dessaisissent les femmes de leur capacité brute de fécondité montraient assez que le moteur de la hiérarchie était là : dans l'appropriation de la fécondité et sa répartition entre les hommes. "

${ }^{63}$ Notamment dans la revue Travail, Genre, Société 10 (2003), p. 173-217.Voir aussi V. Sebillotte Cuchet « La Terre-mère : une lecture par le genre et la rhétorique patriotique ", Kernos 18 (2005), p. 203-218.

${ }^{64} \mathrm{~J}$.W. ScOTT, Gender and the Politics of History, New York, Colombia University Press, $1999^{2}$ (1988) avec une nouvelle préface et un nouveau chapitre : Some More Reflections on Gender and Politics, p. 199-222. Cette préface, ainsi que divers articles publiés depuis, ont été récemment traduits par Claude Servan-Schreiber et publiés dans J.W. ScotT, Théorie critique de l'histoire. Identités, expériences, politiques, Paris, Fayard, 2009.Voir également J.W. SCOTT, «Fantasmes du millénaire : le futur du "genre" au XXI siècle " (2001), Clio HFS 32 (2010), p. 89-117. En 2007, une table-ronde réunissant à Paris Judith Butler, Éric Fassin et Joan W. Scott, donne à cette dernière l'occasion de revenir sur ce débat et d'exprimer son souhait d'un usage toujours critique du mot: «Pour ne pas en finir avec le "genre"...", Sociétés et Représentations 24 (2007), p. 285-306.

${ }^{65}$ Voir par exemple l'usage dit "américain » du gender comme construction culturelle du sexe : M. SkinNER, "Woman and Language in Archaic Greece, or, Why is Sappho a Woman ? ", in N.S. Rabinowitz, A. Richlin (éd.), Feminist Theory and the Classics, New York, 1993, p. 125-144, en part. p. 131-133 où elle distingue oralité et écriture sur le critère du genre et E. STEHLe, Performance and Gender in Ancient Greece, Princeton, Princeton University Press, 1997, qui distingue performance orale (féminine) et mise par écrit (masculine), p. 168-169 et 322-324.

${ }^{66}$ SoHN, « Françoise Héritier », p. 106. 
domination biologique des femmes conduit en effet à sélectionner un aspect du corps parmi d'autres (la matrice ou l'utérus) et une fonction biologique parmi d'autres (la fonction de reproduction) et à généraliser cet aspect et cette fonction à l'ensemble de la perception qu'une société se fait des femmes. On a beau être passé du sexe au genre, reste cette confrontation entre les femmes d'un côté et les hommes de l'autre, deux groupes pensés comme homogènes et opposés. Avec ce présupposé en tête, l'histoire des rapports hommes / femmes et des rapports masculin-féminin, se trouve dans une impasse, condamnée à relever les subtiles variations d'un invariant : la différence des sexes est plus stable que jamais, si structurante qu'elle rend invisible d'autres formes de domination sociale (statut, âge, richesse, etc.).

Il est vrai qu'en insistant régulièrement sur le caractère construit des représentations de genre (ici au sens du féminin et du masculin), Nicole Loraux s'efforce toujours d'éviter le piège qui consisterait à rabattre le genre sur le sexe. Ceci explique d'ailleurs probablement son obstination à refuser le qualificatif de féministe. Les Grecs, ditelle, ont fait du super héros - ici Héraclès - un être également très féminin. Ils ont pensé de la même manière, ajoute-t-elle, le travail en couches des femmes et la mort à la guerre du citoyen. Autrement dit, les Grecs ont pensé le féminin et le masculin comme des caractéristiques non déterminées par le corps, même si elles lui sont étroitement dépendantes ${ }^{67}$. Cette approche, qui se trouvait à l'origine du programme porté par l'outil du genre, comme on l'a dit plus haut, était partagée à la même époque par des collègues états-uniennes, comme Froma Zeitlin, qui travaillait sur la comédie ou Helen Foley sur la tragédie ${ }^{68}$. La plupart empruntait la même direction. John Winkler, par exemple, dans un article important de 1990, montrait que les hommes étaient aussi soumis que les femmes aux contraintes de genre. Les valeurs opposées de l'hoplite et du kinaidos activent la polarité masculin-féminin pour évaluer, dit John Winkler, des comportements masculins ${ }^{69}$. C'est en travaillant

${ }^{67}$ Loraux, Tirésias, p. 29-53 et 142-170.

${ }^{68}$ H.P. Foley, "The Concept of Women in Athenian Drama » et F.I. ZeitLIN, "Travesties of Gender and Genre in Aristophanes' Thesmophoriazousae ", in H.P. Foley (éd.), Reflections of Women in Antiquity, New York - London, 1981, p. 127-168 et 169-218.

${ }^{69}$ J.J. WINKLER, «Faire la loi : la supervision du comportement sexuel dans 
sur la non concordance entre masculin et hommes ou féminin et femmes, en interrogeant les rapports entre discours normatifs et pratiques sociales, et en comparant les places faites aux hommes et aux femmes au sein de même espaces discursifs que l'on est arrivé au renouveau actuel ${ }^{70}$. Pour cela il fallait abandonner la question du "pourquoi la domination masculine ? " pour lui préférer celle $\mathrm{du}$ « comment s'exercent les dominations? »

\section{L'historicisation de la différence des sexes: la pertinence actuelle de l'outil genre}

Histoire des femmes, anthropologie des sexes, histoire du genre, ces différentes formulations signalent la polyphonie d'un courant de recherche dans lequel les approches se tuilent les unes les autres, une voix de dessous pouvant un jour prendre le dessus. L'ambition théorique, affichée dès les années 1980, qui préconisait d'étudier les différentes manières de penser le partage entre le féminin et le masculin, a souvent joué en sourdine, de la même façon que l'histoire de la sexualité qui a d'abord évolué à part. Or c'est en prêtant l'oreille à ces registres que se renouvelle aujourd'hui une histoire du genre orientant son enquête vers les modalités - plus que sur les raisons - de la domination masculine, sans survaloriser la question du sexe.

\subsection{Le bénéfice des études portant sur la sexualité antique}

Inspirées des travaux pionniers de Paul Veyne et de Michel Foucault, les recherches récentes sur la sexualité antique ont montré, depuis les mêmes années 1980-1990, que les sociétés anciennes constituaient un monde d' "avant la sexualité ». En France, les travaux actuels de Sandra Boehringer donnent un accès commode et récent à cette

l'Athènes classique ", in WINKLER, Désirs et contraintes, p. 95-142, en part. p. 105 : « La différence entre l'hoplite et le kinaidos est une opposition entre le mâle masculin et le mâle féminin, et elle repose par conséquent sur une polarité plus fondamentale, celle qui distingue les hommes des femmes " (trad. de S. Boehringer et N. Picard).

${ }^{70}$ Voir aussi la critique apportée par R. Osborne à une lecture trop polarisée des hommes et des femmes dans les actes du culte : R. Osborne, "Women and Sacrifice in Classical Greece ", in R. Buxton (éd.), Oxford Readings in Greek Religion, Oxford, 2000, p. 294-313. 
historiographie et à la déconstruction des catégories contemporaines de la sexualité à laquelle elle a donné $l i u^{71}$. On peut ajouter que le dialogue avec les autres sciences sociales a été, dans ce domaine, particulièrement nourri, en particulier avec les sociologues. Par ailleurs, le rôle moteur joué par les intellectuels militants, gays et lesbiennes en particulier, mérite d'être souligné ${ }^{72}$. Le titre choisi par Froma Zeitlin, John Winkler et David Halperin, Before Sexuality, pour un ouvrage collectif paru en 1990, est éloquent, puisque, ainsi que l'exprime Sandra Boehringer, "dans l'Antiquité gréco-romaine, il n'existe aucun équivalent de cette notion moderne de sexualité ${ }^{73}$.» L'activité du sexe, associée à Éros - puissance du désir - et à Aphrodite, n'est pas perçue indépendamment des autres pratiques du corps, ce qui ne permet, comme le soulignent Florence Dupont et Thierry Eloi, ni de parler d'homosexualité, ni d'hétérosexualité, ni même de bisexualité ${ }^{74}$. Selon Sandra Boehringer,

l'acte sexuel, n'est pas perçu comme un acte concernant conjointement deux partenaires. Les termes latins et grecs exprimant la relation sexuelle, quelle qu'elle soit, déterminent quasiment toujours le rôle assumé dans la relation par l'un et par l'autre, et ces rôles sont très souvent perçus comme différents. Cependant, la désignation de ces rôles par les ter-

${ }^{71}$ S. Boehringer, L'Homosexualité féminine en Grèce et à Rome, Paris, Les Belles Lettres, 2006, en part. p. 28-31; de la même «Sexe, genre, sexualité : mode d'emploi (dans l'Antiquité) ", Kentron, revue du monde antique et de psychologie historique 21 (2006), p. 83-110 et "All'Hagêsichora me teirei (Alcman, fr. 3) : ce que les travaux sur la sexualité apportent aux recherches sur le genre ", in Sebillotte Cuchet, ERnoult, Problèmes du genre, p. 125-145, en part. p. 125-135. Voir également, et en dernier lieu, S. Boehringer, V. Sebillotte Cuchet (éd.), Hommes et femmes dans l'Antiquité grecque et romaine, Paris, Armand Colin, 2011.

${ }^{72}$ D. ERibon, "Le Genre dans les études gays et lesbiennes ", in C. BArd, C. Baudelot, J. Mossuz-Lavau, Quand les femmes s'en mêlent. Genre et pouvoir, Paris, 2004 , p. 247-260, en part. p. 248 : les études gays et lesbiennes «bouleversent la pratique de l'histoire en faisant apparaitre des réalités jusqu'ici ignorées ou négligées, mais aussi, et surtout, en obligeant les historiens à repenser les catégories avec lesquelles ils affrontent le passé et à conceptualiser différemment les réponses qu'ils peuvent apporter. » De ce point de vue, soulignons aussi le rôle joué par les éditions ÉPEL et, notamment, leur collection Les Grands classiques de l'érotologie moderne.

${ }^{73}$ Boehringer, "All'Hagêsichora me teirei », p. 133 ; D. Halperin, J. Winkler, F. Zeituin, Before Sexuality. The Construction of Erotic Experience in the Ancient Greek World, Princeton, Princeton University Press, 1990.Voir aussi, pour Rome, J.P. HaLLett, M.B. Skinner, Roman Sexualities, Princeton, Princeton University Press, 1997.

${ }^{74}$ F. Dupont, T. Élor, L'Érotisme masculin dans la Rome antique, Paris, Belin, 2001. 
mes d" "actif" et de "passif" échoue à rendre compte de la totalité des représentations et de leur complexité ${ }^{75}$.

Divers travaux ont exploré cette question, associée à celle de la compréhension de l'éros antique, parmi eux, ceux de Claude Calame, Froma Zeitlin, Holt Parker ou James Davidson ${ }^{76}$.

De ces études, on conclura, provisoirement (tant les débats sont toujours riches et parfois vifs ${ }^{77}$ ), que pour comprendre la sexualité antique, la notion d'identité sexuelle (au sens d'orientation sexuelle) n'est pas d'un grand secours : en effet, le sexe n'est pas le critère qui permet de classer et d'évaluer la sexualité des individus. La classification se fait sur le critère du convenable ou du non convenable, du kata nomon ou du para nomon. Par ailleurs, les chercheuses ont montré que c'était presque toujours la sexualité considérée du point de vue des hommes qui était en jeu, tout simplement parce que la sexualité entre femmes ne s'exprime pas dans le champ où on l'attend ${ }^{78}$.

La reproduction - qui est pour nous la forme la plus commune sous laquelle nous pensons la sexualité - n'est pas, du fait de ses conséquences politiques en termes de transmission de patrimoine et de statut, prioritairement perçue par les Grecs comme relevant du domaine d'Éros. Cela ne signifie pas que différentes fonctions biologiques et sociales, telles que celles mises en jeu dans le mariage, ne puissent lui être associées. Néanmoins, puisque la différence des sexes ne structure pas les conduites en matière de gestion des plaisirs, il est tout à fait légitime de poser la question de sa pertinence dans d'autres domaines de la vie sociale. En ce sens, oui,

75 Boehringer, "All'Hagêsichora me teirei », p. 133.

${ }^{76}$ C. Calame, L'Éros dans la Grèce antique, Paris, Belin, $2009^{2}$ (1996), p. 184194 ; F.I. Zeituin, « Reflections on Erotic Desire in Archaic and Classical Greece », in J.I. Porter (éd.), Constructions of the Classical Body, Ann Arbor, 2005 (1999), p. 50-76 ; J.N. DAvidDSON, « Dover, Foucault and Greek Homosexuality: Penetration and the Thruth of Sex ", Past and Present 170 (2001), p. 3-51 ; H.N. Parker, "The Myth of the Heterosexual: Anthropology and Sexuality for Classicists ", Arethusa 34/3 (2001), p. 313-362, en part. p. 341.

${ }^{77}$ Voir à ce propos les échanges publiés en ligne par la Bryn Mawr Classical Review (http://bmcr.brynmawr.edu).

${ }^{78}$ Voir P. Du BoIs, Sowing the Body. Psychoanalysis and Ancient Representations of Women, Chicago - London, The University of Chicago Press, 1988 ; BoeHRINGER, L'Homosexualité feminine, p. 361-363 et l'hommage qu'elle rend (p. 23-24) à la production des chercheuses américaines. 
ainsi que le souligne Sandra Boehringer, les études sur la sexualité font avancer la recherche sur le genre.

\subsection{Les apports de l'histoire}

des discours techniques et médicaux sur le sexe

Les conclusions des travaux sur la sexualité convergent avec celles de Thomas Laqueur qui, le premier, me semble-t-il, a historicisé le sexe. C'est au XVIII siècle, explique Thomas Laqueur, que se développe un discours sur les deux sexes : le développement de la science anatomique isole le corps des actes qu'il accomplit ainsi que de son environnement social. Avant l'époque moderne, poursuit Thomas Laqueur, les femmes et les hommes se construisaient socialement, c'est-à-dire en tant que femmes ou hommes, par l'intermédiaire de rites accomplis en particulier lors de la puberté. Être femme et être homme n'apparaissaient pas comme une donnée de naissance mais comme une lente fabrication, un accomplissement social, ce que Laqueur désigne par le terme de genre.

Pour aller en ce sens, on peut-on ajouter que le terme de sexe n'existe pas en grec comme catégorie générale désignant ce que nous nommons le « sexe " en français - c'est-à-dire la différence constitutive du mâle et de la femelle dans les animaux et dans les plantes - autrement dit l'organe - ou " collectivement les hommes ou les femmes ${ }^{79} »$. Des termes existent en grec pour désigner les hommes comme individus mâles (arrên) et d'autres les femmes en tant que femelles (thêlu), mais, selon Sandra Boehringer, « ces termes sont spécifiques pour chacune des catégories et très rarement employés de manière symétrique ${ }^{80}$. " Le sexe n'est pas un critère abstrait de différenciation, il est un élément d'une réalité concrète et diverse. Pour parler des organes génitaux, les Grecs emploient les termes d'aidoia ou de phusis, lesquels n'ont pas non plus de valeur générale. C'est du latin sexus que vient notre mot sexe. Comme en grec, le mot n'est pas utilisé de manière abstraite

${ }^{79}$ Telles sont les deux définitions données par le Littré et toujours valables ; la troisième, "le beau sexe, absolument le sexe, les femmes ", l'est moins.

${ }^{80}$ S. BOEHRINGER, "Un autre genre d'amour? De quelques mouvements du désir dans la poésie grecque et romaine ", Champ psychosomatique 58 (2010), p. $83-105$, en part. p. 84 . 
et générale, il est généralement assorti d'un adjectif spécifiant s'il s'agit du sexus virile ou du sexus muliebre.

Ce qui distingue les individus c'est donc leur genre, leur catégorie sociale qui mêle éléments biologiques et sociaux : être un homme (anêr, vir) au sens d'adulte citoyen pourvu de qualités morales spécifiques, être une femme (gunê, mulier) au sens d'adulte citoyenne pourvues de qualités morales spécifique, être un enfant (pais, puer), au sens d'individu en cours de maturation du point de vue du genre, etc. Des individus qui, à nos yeux, auraient un genre manifestement différent de leur sexe, ne posaient pas de problème si l'on en croit l'auteur du traité Du Régime : on peut être de sexe féminin et de tempérament audacieux, on sera qualifié de virile (andreiai), et on peut être de sexe masculin et qualifié d'androgyne (androgunoi) sans que ces deux adjectifs n'aient de connotation dépréciative. Le genre, masculin ou féminin, est ici conçu comme une caractéristique autonome et indépendante du sexe de l'individu qu'il caractérise. Le genre signifie simplement, pour la première, que la semence femelle qui a produit le sexe féminin provient du père seul, pour le second, que la semence mâle qui a produit le sexe masculin provient de la mère seule ${ }^{81}$. Des peuples entiers (constitués par définition d'hommes et de femmes) peuvent s'adonner à des occupations comme la musique ou le commerce qui les détournent de la guerre et leur valent la caractérisation de "féminin » : le genre institue ici une hiérarchie entre des peuples, il est indépendant du sexe des individus concernés ${ }^{82}$.

La thèse de Laqueur a donné lieu à de nombreuses critiques portant sur le choix de la césure du XVIII ${ }^{\mathrm{e}}$ siècle, sur la simplification des «modèles » dans la manière de penser le corps, sur la survalorisation d'un unique modèle pré-moderne, le modèle "unisexe " (qui est en fait surtout galénique), ainsi que sur la survalorisation des discours des anatomistes du XIX ${ }^{\mathrm{e}}$ siècle pour caractériser la

${ }^{81}$ Hippocrate, De diaeta, I, 28-29 = R. Joly, Hippocrate : Du Régime, Paris, Les Belles Lettres, C.U.F., $2003^{2}(1967)$.

${ }^{82}$ Hérodote, Historiae, I, $155=$ Ph.-E. Legrand, Hérodote $:$ Histoires, Paris, Les Belles Lettres, C.U.F., $1993^{6}$ (1932). Dans un autre champ discursif, celui des physiognomonistes et sophistes du $\mathrm{II}^{\mathrm{e}}$ siècle de notre ère, tels Polémon ou Favorinus, Maud Gleason analyse la construction des bonnes et mauvaises masculinités à travers l'usage de polarités exprimées en terme d'opposition masculin-féminin : M.W. Gleason, Making Men: Sophists and Self-Presentation in Ancient Rome, Princeton, Princeton University Press, 1995. 
période moderne. On lui a reproché de confondre la biologie avec la "nature " et de ne pas prendre en compte la dimension politique de la lecture biologique des corps faite par les médecins à partir du XIX ${ }^{\mathrm{e}}$ siècle. Enfin on lui a également reproché de laisser croire que les discours scientifiques pouvaient à eux seuls causer des changements majeurs dans les représentations sociales ${ }^{83}$.

En suscitant la controverse, cette thèse a contribué à ce que le sexe et la différence des sexes fassent l'objet d'enquêtes de plus en plus précises qui, aujourd'hui, soulignent la diversité des manières de penser le corps féminin et le corps masculin, y compris dans l'Antiquité. Si la plupart des traités médicaux et biologiques antiques insistent sur la dépendance du corps de la femme envers sa nature, constituée par des humeurs froides et humides et un organe instable, imparfaite par rapport au corps de l'homme, cette représentation n'affecte pas l'ensemble de la vie sociale. Elle est surtout liée aux enjeux poursuivis par de tels traités : expliquer la dépendance des femmes envers la fonction de reproduction ${ }^{84}$. Il y a donc, dans les représentations que les Anciens se font du corps des femmes et de celui des hommes, à la fois du genre et du sexe, du biologique et du social.

Depuis, et même avec les nuances et les correctifs nécessaires apportés à la thèse de Thomas Laqueur, on ne peut plus penser le sexe comme un invariant biologique qui serait porteur de déterminations psychiques spécifiques : c'est toujours dans le cadre d'enjeux sociaux particuliers (dans un espace discursif particulier) que la différence des sexes est formulée. Dès lors, il n'y a plus aucune raison de privilégier l'idée que les philosophes ou les médecins antiques se sont fait du sexe (un espace discursif parmi d'autres) pour réfléchir aux relations entre les hommes et les femmes, aux identités de sexe et aux rapports de sexe. Chaque espace discursif, avec les pratiques qui lui sont propres, peut produire un type de représentation particulier, une logique de genre particulière.

${ }^{83}$ Pour ces critiques voir E. Dorlin, "Autopsie du sexe ", Présences de Simone de Beauvoir, Les Temps modernes 619 (2002), p. 115-143, ainsi que S. STEINBERG, "Sexe et genre au XVIII siècle. Quelques remarques sur l'hypothèse d'une fabrique du sexe ", in Théry, Bonnemère, Ce que le Genre fait aux personnes, p. 197-212.

${ }^{84}$ H. King, Hippocrate's Woman. Reading the Female Body in Ancient Greece, London - New York, Routledge, 1998 ; L. Dean-Jones, Women's Bodies in Classical Greek Science, Oxford, Clarendon Press, 1996. 


\subsection{Pour en finir avec les femmes}

Une nouvelle méthode, celle de la comparaison systématique des situations des hommes et des femmes dans des espaces discursifs particuliers a été proposée dans les années 2000 par Pauline Schmitt Pantel, dans le cadre d'un programme de recherche collectif sur le genre dans l'Antiquitée ${ }^{85}$. Cette méthode a été mise en ouvre dans différents travaux ${ }^{86}$.Je ne prendrai ici que quelques exemples des résultats obtenus.

En travaillant sur la représentation des hommes et des femmes sur les images de banquet, P. Schmitt Pantel a retrouvé, sans grande surprise, la distinction classique depuis les travaux sur la sexualité antique, des individus selon un double critère, de statut et d'âge : "La hiérarchie est certes présente au banquet, dit-elle, mais elle sépare à mon sens les hommes adultes qui mènent le jeu et les autres, hommes jeunes et femmes ${ }^{87}$.» En étudiant les gestes accomplis dans les sanctuaires, elle observe, par ailleurs, que des hommes et des femmes lavent les statues des dieux, les habillent et les décorent de la même façon. Elle conclut : «Quand nous disposons d'indices, aucune règle, aucun partage ne peut être repéré entre les hommes et les femmes ${ }^{88}$. " De même, s'intéressant au partage des espaces, elle remarque que ceux-là ont trop rapidement été découpés par les historiens et historiennes en "masculins » et " féminins ». Dans un article de synthèse intitulé "Des espaces partagés ", Pauline Schmitt Pantel recense d'autres travaux récents qui concluent également à la nécessité d'articuler la question de la différence des sexes à d'autres caractéristiques sociales, comme le statut dans la

${ }^{85}$ Ce programme était inscrit dans les axes de recherche de l'EA 3521 Phéacie, Pratiques culturelles des sociétés grecque et romaine, Université Paris I et Université Paris VII (2001-2009), désormais intégrée au sein de l'UMR 8210 ANHIMA (Anthropologie et Histoire des Mondes Antiques), INHA, 2 rue Vivienne, Paris.

${ }^{86}$ Pour l'essentiel, c'est la méthode adoptée par les participants à l'ouvrage collectif Sebillotte Cuchet, Ernoult, Problèmes du genre.

${ }^{87}$ Schmitt Pantel, Aithra et Pandora, p. 145-157, en part. p. 157, renouvelant un article publié en 2003 : «Le Banquet et le "genre" sur les images grecques, propos sur les compagnes et les compagnons ", Pallas 61 (2003) (Symposium, banquet et représentation en Grèce et à Rome, colloque international, Université de Toulouse Le Mirail, 7-9 mars 2002), p. 83-95.

${ }^{88}$ Schmitt Pantel, Aithra et Pandora, p. 112-122, en part. p. 12, reprenant un article de $2008:$ : La Manipulation rituelle des images grecques étudiée sous l'angle du genre ", in S. Estienne, D. JAillard, N. LubTCHAnSKy (éd.), Image et religion dans l'Antiquité gréco-romaine, Napoli, 2008, p. 307-314. 
cité et/ou dans la parenté (en particulier lors des dépôts d'objets dans les tombes ou la circulation des personnes dans les pièces de la maison $)^{89}$.

Dans l'espace discursif du patriotisme, et intégrant les discours qui peuvent parodier la rhétorique patriotique, j'ai moi-même montré que les filles et les garçons sont considérés comme faisant partie du même groupe de personnes, celles qui sont intimement concernées par la défense de la cité. Elles s'opposent, à des degrés divers, à la fois aux étrangers et aux esclaves, mais aussi aux citoyens plus âgés, hommes et femmes, qui, responsables de leur propre oikos, ne peuvent pas développer une attitude de fidélité aussi exigeante et radicale à l'oikos commun (la polis) que les jeunes. La " virginité " (partheneia) des filles est la manière qu'ont les Athéniens d'exprimer leur fidélité à l'oikos, cette partheneia étant elle-même métaphore de la fidélité des fils à la patrie civique et de leur engagement consécutif dans la guerre de défense du territoire ${ }^{90}$.

Dans l'espace discursif du polythéisme, Gabriella Pironti a montré qu'Aphrodite, divinité qui relève autant de la guerre que de l'érotisme, intervient dans des domaines qui ne sont ni "féminins " ni spécifiquement réservés aux femmes, c'est le moins que l'on puisse dire $^{91}$. De fait, les divinités ne sont pas caractérisées, en premier lieu, par leur genre ${ }^{92}$.

Ces récents travaux aboutissent à dessiner une cartographie du genre où la différence des sexes apparaît plus ou moins signifiante. Les domaines dans lesquels la différence des sexes est la plus opérante sont, évidemment, ceux dont l'enjeu est de décrire la reproduction humaine. Aujourd'hui une approche de la recherche pourrait consister à délimiter les lieux dans lesquels la différence des sexes

${ }^{89}$ Schmitt Pantel, Aithra et Pandora, p. 105-109, évoquant les travaux de M. Cuozzo, Reinventando la tradizione. Immaginario sociale, ideologie e rappresentazione nelle necropoli orientalizzanti di Pontecagnano, Paestum, Pandemos, 2003 et ceux de L. Nevett, House and Society in the Ancient Greek World, Cambridge, Cambridge University Press, 1999.

${ }^{90}$ V. Sebillotte Cuchet, «La Sexualité, une histoire problématique pour les hellénistes. Détour par la "virginité" des filles sacrifiées pour la patrie ", Mètis n.s. 2 (2004), p. 137-161 et EAD., Libérez la patrie! Patriotisme et politique en Grèce ancienne, Paris, Belin, 2006, p. 291-317.

${ }^{91}$ G. Pironti, Entre ciel et guerre. Figures d'Aphrodite en Grèce ancienne, Liège, Centre international d'étude de la religion grecque antique, 2007 (Kernos, suppl. 18).

${ }^{92}$ N. Loraux, "Qu'est-ce qu'une déesse ? ", in Schmitt Pantel, Histoire des femmes, p. 31-62. 
est opératoire et les modalités de son impact social en général. Dans cette perspective, le domaine dit « du politique » est particulièrement intéressant. Selon Pauline Schmitt, le politique est l'espace par excellence qui exclut les femmes, celui qui rend la différence des sexes particulièrement nette ${ }^{93}$. Or, si l'on suit Josine Blok, les femmes sont totalement incluses dans la citoyenneté, conçue, comme le politique, comme une alliance collective (de maisons) conclue entre la polis et ses dieux. Si l'on admet que la rhétorique patriotique fait des citoyennes et de leurs filles des actrices indispensables à la bonne marche de la polis, que les héroïnes sont, dans ce contexte, aussi remarquables que les hommes citoyens, c'est d'un aspect très particulier du politique (qu'il reste à circonscrire précisément) qu'il faut parler lorsque l'on évoque l'exclusion des femmes ${ }^{94}$.

La question est de savoir si nous privilégions le filtre contemporain (la domination masculine), le filtre biologique (la pensée des deux sexes produite par certains discours médicaux du XIX siècle) ou le filtre de l'anthropologie du politique (le politique gouverne la cité et, d'une manière ou d'une autre, ce politique renvoie à une conception de la citoyenneté uniquement masculine) pour comprendre le monde grec antique. Si l'analyse des sources propres à chaque espace discursif ne valide pas systématiquement la hiérarchie sexuelle mais qu'elle distingue les individus sur d'autres critères, c'est que la différence des sexes n'est pas la structure organisatrice par excellence de la cité grecque ${ }^{95}$. L'enjeu des études sur le genre, entendu désormais comme interrogation sur la part prise par la différence des sexes dans les sociétés, est de déterminer où, quand et comment la différence des sexes est significative.

De ce point de vue, il est important de reconsidérer la lecture habituelle que les Antiquistes font de Pandora, désignée par eux comme "la première femme ", ou l'ancêtre de "la race des femmes ${ }^{96} »$. On oublie trop souvent que le terme utilisé par le poète,

${ }^{93}$ Schmitt Pantel, Aithra et Pandora, p. 29, 36 et supra note 30.

${ }^{94}$ Supra note 30 et voir V. Sebillotte Cuchet, « Clio a lu », Clio HFS 30 (2009), p. 256.

${ }^{95}$ Les études sur la parenté montrent, par exemple, comment la filiation (genos) articule des données liées au sexe, à l'âge et à l'alliance : voir notamment A. BRESSOn, M.-P. Masson, S. Perentidis et alii (éd.), Parenté et société dans le monde grec de l'Antiquité à l'âge moderne, Bordeaux, Ausonius, 2006 (Études, 12).

${ }^{96}$ Loraux, Les Enfants d'Athéna, p. 75-117, en part. p. 78. 
gunê ${ }^{97}$, désigne très souvent une réalité bien plus précise : celle de l'épouse légitime. Pandora n'est pas l'ancêtre des femmes car les femmes comme catégorie homogène n'existent pas. Seules existent des esclaves, des étrangères, des vendeuses de rubans, des mères et des filles, des Athéniennes et des Perses, des épouses et des compagnes $^{98}$. La Pandora hésiodique n'est qu'une des figures mythiques de la déchéance des mortels, qui se sont progressivement éloignés du mode de vie divin ${ }^{99}$. Dans ce cadre là, elle exprime la nouvelle nécessité de la reproduction légitime pour qui veut transmettre sa terre, le fruit de son travail et se donner, à travers le fils héritier, un semblant d'immortalité ${ }^{100}$. Elle exprime la désolation d'une vie conjugale imposée par la nécessité de la transmission du patrimoine dans le cadre de l'oikos légitime. Si la grâce habille Pandora et trompe son futur mari - un malheureux ${ }^{101}$ - c'est qu'Aphrodite participe au piège tendu par Zeus pour punir l'humanité, un piège qui vise à la fois des hommes et des femmes, tous ceux qui ont quelque chose à transmettre.

Si tous les conseils donnés par le poète à son auditoire entretiennent l'idée d'une opposition radicale entre l'homme et la femme (anêr et gunê) de même qu'entre fille et garçon ${ }^{102}$, ces différences s'inscrivent dans un même contexte : celui d'une société organisée autour de la production et de la reproduction. Ce sont ces thématiques seules qui donnent sens à la différence des sexes, une différence des sexes qui s'inscrit à l'intérieur de deux césures bien plus fondamentales, car qui la conditionnent, celle qui sépare mortels et

${ }^{97}$ Hésiode, Theogonia, 551-616 (en part. 590) et Opera et dies, 42-105 = P. Mazon, Hésiode : Théogonie - Les Travaux et les jours - Le Bouclier, Paris, Les Belles Lettres, C.U.F., $1992^{13}$ (1928). La bibliographie, depuis les travaux de J.-P.Vernant et de F. Zeitlin, est extrêmement importante.Voir en dernier lieu : Schmitt Pantel, Aithra et Pandora, p. 195-215.

${ }^{98}$ La diversité des femmes était un point que Claude Mossé avait relevé comme n'étant pas suffisamment pris en compte par l'entreprise de l'Histoire des femmes en Occident : Mossé, « L'Antiquité », p. 21.

${ }^{99}$ Hésiode, Theogonia, 570-612. Sur les modalités de la différenciation : D. SAINTILlan, « Du festin à l'échange : les grâces de Pandora », in F. Blaise (éd.), Le Métier du Mythe. Lectures d'Hésiode, Lille, 1996, p. 315-348.

${ }^{100}$ Hésiode, Opera et dies, 376, 607.

${ }^{101}$ Hésiode, Opera et dies, 84. Sur la grâce de Pandora : Hésiode, Theogonia, 605-606.

${ }^{102}$ Par exemple, les bains doivent être pris séparément, les dates convenables pour donner le jour à une fille ne sont pas les mêmes que celles qui sont convenables pour donner le jour à un garçon : HÉsIODE, Opera et dies, 753, 779, 783-785. 
immortels et celle qui séparent propriétaires et non-propriétaires. Le piège n'est pas la femme, ni les femmes comme groupe déjà constitué, mais le désir de transmission, le désir d'une filiation légitime, ce désir qui entraîne la nécessité du couple légitime ${ }^{103}$.

Une anthropologie de l'Antiquité en prise avec son présent ne peut laisser de côté l'apport très significatif des recherches intéressant l'ensemble des sciences sociales, irriguées par les revendications puis les théories féministes. L'étude de l'Antiquité est soumise à la médiation de documents élaborés dans des contextes où, bien souvent, les questions sur l'identité de sexe, la différence de sexe ou les rapports de sexe ne se posaient pas. À extraire les seules sources qui parlent du sexe, le risque est la survalorisation d'un espace discursif particulier et, de ce fait, d'une logique de genre particulière (c'est-à-dire une manière de disposer les hommes et les femmes selon des enjeux qui sont propres à son champ discursif). On pense en premier lieu à la mise en avant des discours techniques de nature médicale ou biologique. En étendant la recherche à tous les espaces discursifs de la vie sociale, la démarche propose d'enquêter sur les moments, les espaces, les manières avec lesquelles la différence des sexes intervient, discrimine ou module une césure qui se fonde très souvent sur un autre critère.

Si la question du genre, entendu comme questionnement sur la place accordée à la différence des sexes, est une question contemporaine et totalement anachronique dans les sociétés antiques, elle mérite néanmoins, en raison des bienfaits incontestables d'une méthode défendue en son temps par Nicole Loraux ${ }^{104}$, d'être posée. Les réponses apportées par des recherches portant sur les sociétés anciennes - comme souvent celles apportées par l'étude des sociétés non-occidentales - font toujours mieux apparaitre les rigidités de notre présent qui, en l'occurrence, a tendance à généraliser la différence des sexes à tous les aspects de la vie sociale, sans compter qu'elles renouvellent, par l'introduction de la mixité dans l'écriture de l'histoire, des pans entiers de notre connaissance de l'Antiquité.

${ }^{103}$ Cette lecture du mythe de Pandora a été exposée, avec davantage d'arguments, dans mon mémoire d'HDR (encore inédit), Artémise, l'Amazone qui a existé, soutenue le 14 novembre 2009 à l'université de Paris I.

104 N. LORAUX, «Éloge de l'anachronisme en histoire ", Le Genre humain 27 (1993), p. 23-39. 